Nouvelles perspectives en sciences sociales

Revue internationale de systémique complexe et d'études relationnelles

\title{
De la naturopathie rurale à la santé naturelle : distanciation et assimilation autour de la notion d'espace
}

\section{Anahita Grisoni}

Volume 8, numéro 1, novembre 2012

URI : https://id.erudit.org/iderudit/1013924ar

DOI : https://doi.org/10.7202/1013924ar

Aller au sommaire du numéro

Éditeur(s)

Prise de parole

ISSN

1712-8307 (imprimé)

1918-7475 (numérique)

Découvrir la revue

Citer cet article

Grisoni, A. (2012). De la naturopathie rurale à la santé naturelle : distanciation et assimilation autour de la notion d'espace. Nouvelles perspectives en sciences sociales, 8(1), 237-259. https://doi.org/10.7202/1013924ar 


\title{
De la naturopathie rurale à la santé naturelle : distanciation et assimilation autour de la notion d'espace
}

\author{
Anahita Grisoni \\ École des hautes études en sciences sociales, Centre d'analyse et \\ d'intervention sociologiques (CADIS), Paris
}

\section{Introduction}

T a naturopathie est une médecine douce, basée sur le " ren- forcement de l'énergie vitale de l'homme par des moyens naturels ${ }^{1} »$. Elle repose essentiellement sur des techniques de soins en rapport avec l'alimentation, et prône un régime basé sur des ingrédients issus de l'agriculture biologique. Dans le contexte d'une montée des pratiques liées à la consommation critique ${ }^{2}$, le fort engouement pour les produits bio $^{3}$ et le commerce équitable ${ }^{4}$, la naturopathie trouve un terrain favorable pour se développer sur la nébuleuse écologico-environnementale et au-delà.

\footnotetext{
$\overline{1}$ Définition de la Fédération Française de Naturopathie (FENAHMAN).

2 Geoffrey Pleyers (dir.), La Consommation critique. Mouvements pour une alimentation responsable et solidaire, Paris, Desclée de Brouwer, 2010.

3 Claire Lamine, Les Intermittents du bio, pour une sociologie pragmatique des choix alimentaires émergents, Paris, La Maison des Sciences de l'Homme, 2008.

4 Virginie Diaz Pedregal, Le Commerce équitable dans la France contemporaine, Paris, L'Harmattan, 2008.

5 André Micoud, "De l'expert-militant à l'être vivant sensible », dans Marcel
} 
Cette thérapie connaît aujourd'hui un processus de transformation qui, loin de concerner seulement la discipline, tend à modifier les pratiques et les représentations liées à l'alimentation, au corps et au bien-être dans les domaines de la santé et de l'environnement. Cette évolution reflète une tension entre deux manières de pratiquer la naturopathie, basées sur des techniques de soins, mais aussi des discours et des représentations, très divergentes: la naturopathie rurale et la naturopathie urbaine. Davantage qu'une question de génération, c'est le mode de vie rural ou urbain qui marque les frontières entre une naturopathie qui se voudrait orthodoxe, vitaliste, dans la continuité du travail du fondateur de la discipline en France, et une pratique urbaine, liée à l'essor des problématiques liées au couple santé/environnement, qui touchent une catégorie de personnes tout à fait nouvelle. Ainsi, les néo-ruraux des années 1970 deviennent des clients, la contre-culture contestataire s'efface au profit des pratiques de consommation critique. Et les logiques d'inclusion et d'exclusion propres à cette discipline passent avant tout par le prisme de la localisation. Si l'ensemble des praticiens formés à l'école de Marchesseau sont passés par des expériences communautaires rurales, il n'en demeure pas moins qu'aujourd'hui, la visibilité de la naturopathie est surtout urbaine.

Cet article est tiré d'une thèse réalisée sur la profession de naturopathe en région parisienne entre 2006 et 2011. Ce travail de thèse est composé de soixante-dix entretiens semi-directifs, menés auprès des clients, des élèves et des professionnels de la naturopathie. Cette dernière catégorie concerne les personnes qui, dans un second temps de leur vie professionnelle, suivent une formation dans une école de naturopathie, suite à laquelle ils ouvrent un cabinet, deviennent enseignant et/ou investissent dans un magasin d'alimentation bio. La plupart d'entre eux viennent initialement des métiers de la communication ou du domaine médical ou paramédical : infirmières, pharmaciens,

Boyer, Guy Herzlich et Bruno Maresca (dir.), L'Environnement, question sociale. Dix ans de recherches pour le ministère de l'Environnement, Paris, Odile Jacob, 2001 p. 121-134. 
kinésithérapeutes, etc. Dans la mesure où la naturopathie n'est pas reconnue par l'État, ils ont un statut de profession libérale. Les entretiens semi-directifs étaient basés sur la narration du récit de vie, à travers l'évolution des techniques de soins et/ou du parcours professionnel. Le travail d'enquête a été complété par trois ans d'observation participante dans les associations professionnelles de naturopathie, dans une association humanitaire dédiée à la santé naturelle, et auprès des praticiens de santé naturopathes. Tous les entretiens ont été menés à Paris ou en région parisienne lorsque les thérapeutes y avaient un cabinet de consultation. Les naturopathes orthodoxes, majoritairement installés en milieu rural, ont été rencontrés et interrogés lors des salons nature et santé ${ }^{6}$ réunissant les professionnels plusieurs fois par an dans la capitale. Les entretiens présentés dans l'article ont été choisis parce qu'ils étaient particulièrement caractéristique de l'un ou de l'autre des deux profils de naturopathes en tension, développés plus loin dans l'article.

Pourtant, si des médecines douces telles que l'acupuncture ou l'ostéopathie bénéficient d'une reconnaissance juridique et sont présentes dans les institutions de santé publique comme dans les organismes de santé privée ${ }^{7}$, le statut légal de la naturopathie est celui d'une prestation de service. Les origines religieuses de cette

$6 \quad$ L'expression " salon nature et santé » désigne l'ensemble des salons commercialisant des produits relatifs aux médecines douces, à l'alimentation et aux cosmétiques issus de l'agriculture biologique et présentant des associations auto-définies comme associations écologiques. À ce titre, on peut citer le salon Marjolaine, le salon zen, le salon des médecines douces, le salon Ecobat, le salon Naturally. Les nominations et les dates varient selon les années, mais une seule société, la société de communication et d'événementiel S-pas, dirigée par l'ancien président de l'ONG Greenpeace en France, assure la gestion de tous ces événements. D'un salon sur l'autre, on retrouve les naturopathes exposants ou employés par les marques d'aliments et de cosmétiques bio.

7 La thèse d'Eliane Bailly, Les Ostéopathes face à la contrainte institutionnelle : genèse d'une professionnalisation, thèse de sociologie soutenue à l'université de Nantes, en 2006, portant sur la reconnaissance juridique de l'ostéopathie, constitue une exception, en intégrant la dimension conflictuelle de ce processus. 
" posture thérapeutique ${ }^{8}$ " inscrite dans la mouvance New Age ont pour conséquence une forte suspicion des institutions de santé publique, relativement au caractère non rationnel-scientifique des techniques de soins qu'elle propose et du rapport instauré entre le thérapeute et le client. Comme souvent dans le cas des médecines douces, c'est l'accusation de secte qui pèse sur les praticiens ${ }^{10}$. Cette méfiance est notamment illustrée par les derniers rapports produits par la Mission Interministérielle de Lutte contre les Dérives Sectaires (Miviludes), qui mettent l'accent sur les " risques " de dérives sectaires que représentent les structures de promotion de l'alimentation naturelle. C'est pourquoi les " praticiens de santé naturopathes ${ }^{11}$ développent leur thérapie en étant particulièrement attentifs aux éventuelles dénonciations et procès engagés par les ordres professionnels médicaux et paramédicaux.

Ce double constat fait donc état d'une tension : d'un côté la naturopathie semble accompagner le consensus croissant autour de la protection de l'environnement ${ }^{12}$ et de la consommation critique $^{13}$ dans la société française contemporaine; de l'autre elle fait l'objet de toutes les suspicions en ramenant la question religieuse au cour du domaine de la santé. Aujourd'hui, cette tension est à l'origine d'une reformulation des groupes d'acteurs, suivant qu'ils situent leurs pratiques et leurs représentations du

$8 \quad$ Anahita Grisoni, Sous les pavés la terre : entre culte du bien-être et nouveaux métiers : la naturopathie en transformation à la conquête de l'espace public, thèse de doctorat en sociologie, 2011, École des hautes études en sciences sociales, Paris.

9 Gilles Tétard, Le Sang des fleurs, une anthropologie de l'abeille et du miel, Paris, Odile Jacob, 2005.

10 Frédéric Lenoir, Nathalie Luca, Sectes, mensonges et idéaux, Paris, Bayard, 1997.

11 Le terme de " praticien de santé naturopathe » est celui qui est mis en avant par les directeurs de centre de formation en naturopathie dépendants de la FENAHMAN, relativement à la profession de naturopathe, non reconnue en France. Le diplôme de praticien de santé naturopathe est délivré à l'issue d'un examen national organisé par la fédération.

12 Florence Rudolf, Le Climat change... et la société?, Paris, Éditions Engagé-e-s, 2008.

13 Ibid., p. 1. 
côté de la naturopathie classique ou des formes contemporaines que prend la discipline.

Dans ce contexte, une partie des clients, des élèves et des professionnels s'est engagée dans une lutte pour la reconnaissance officielle de la naturopathie comme thérapie. À travers la mise en place d'un réseau national d'associations loi 1901, dont les raisons sociales sont aussi variées que le regroupement professionnel, la communication interne et externe, la promotion de l'alimentation biologique, les praticiens de santé naturopathes réalisent un travail de standardisation des connaissances, de la transmission et de l'évaluation de la discipline dans le double objectif d'obtenir la reconnaissance légale de la naturopathie et de pouvoir intégrer les institutions de la santé publique. Dès lors, la naturopathie apparait comme un "nouveau mouvement social ${ }^{14}$ ", croisant les acteurs de la nébuleuse écologico-environnementale ${ }^{15}$, épousant des revendications culturelles, portant sur le mode de vie et se fixant comme cadre le " culte du bien-être ${ }^{16}$ " à travers la quête de la "santé parfaite ${ }^{17}$ ".

Cette nouvelle dynamique d'adaptation aux normes en matière de santé n'est pas sans créer de heurts dans le milieu des naturopathes. Pour comprendre la volonté appuyant le processus de distanciation d'une partie des professionnels, il est nécessaire de considérer les dynamiques mémorielles sous-jacentes qui caractérisent cette discipline. D'un côté, les nouveaux professionnels de la naturopathie tendent à se distancier des techniques de soins, du discours, des pratiques par trop évidemment inscrites dans la mouvance religieuse New Age ${ }^{18}$; de l'autre, le marché de la santé ${ }^{19}$ leur permet de s'assimiler, d'intégrer et de modifier, chaque jour davantage, les pratiques alimentaires et les pratiques de soins de l'ensemble de la société. Nous verrons dans cet article que la

\footnotetext{
14 Alain Touraine et al., La Prophétie anti-nucléaire, Paris, Seuil, 1980.

15 Ibid., p. 1.

16 Ibid., p. 3.

17 Lucien Sfez, La Santé parfaite, Paris, Seuil, 1995.

18 Enzo Pace, "Le Retour du sacré ", dans James Andrew Beckford et Danièle Hervieu Léger (dir.), Religion et Écologie, Paris, Le Cerf, 1993, p. 197-213.

19 Olivier Faure, Histoire sociale de la médecine (18 ime-20 ime siècle), Paris, Anthropos, 1994.
} 
réappropriation de la mémoire des acteurs et des techniques de soins de la naturopathie constitue un enjeu pour la reconnaissance juridique de la discipline.

La notion d'espace, prise comme objet et comme sujet pour la sociologie est particulièrement fertile pour expliquer ce double mouvement de distanciation et d'assimilation. Cette notion sera en partie appréhendée à partir du récit oral et écrit que les professionnels de la naturopathie ont fait de la mémoire de la discipline. Cinq ans d'enquête de terrain auprès des thérapeutes et des clients de la naturopathie m'ont permis de mettre en évidence le passage de la naturopathie d'espaces contre-culturels, communautaires et néo- ruraux $^{20}$ à un espace urbain et commercial. Cette évolution, loin d'être uniquement géographique, concerne en réalité le choix d'un mode de vie. Dans le contexte communautaire des années 1960 et 1970, les pratiques de "santé naturelle " représentaient l'une des dimensions d'une posture contestataire incarnée par un ensemble de pratiques basé sur la déviance et imprégné de religiosité New $\mathrm{Age}^{21}$. Progressivement, la spécialisation de la naturopathie et l'émergence de centres de soins et de formation sur le territoire urbain ont généré une division entre deux types de naturopathies, évoluant sur deux types d'espaces, entre deux modes de conception religieuse. Discipline secondaire, aux évolutions d'échelle microsociologique, la naturopathie est néanmoins inscrite dans un processus d'évolution et de diffusion des pratiques et de représentations liant la santé à l'environnement sur l'espace commercial. Elle sera donc appréhendée ici comme un élément d'explication aux phénomènes plus amples, aux contours flous, de changement des modèles en termes de techniques du corps, d'alimentation et de représentation sociale de la nature ${ }^{22}$.

Différenciation et homogénéisation sont donc deux processus interdépendants qui sont le moteur de la naturopathie contem-

\footnotetext{
20 Bernard Hervieu et Danièle Léger, Le Retour à la nature, au fond de la forêt... l'État, Paris, Seuil, 1979.

21 Paul Heelas, The New Age Movement, Londres, Blackwell Publishers, 1996.

22 Philippe Descola, Par-delà nature et culture, Bibliothèque des sciences humaines, Gallimard, Paris, 2005.
} 
poraine. Mettre l'étude du passage d'un type d'espace à un autre au cour de la démarche théorique et sociologique permet de contourner l'analyse linéaire de la simple sécularisation ou adaptation de la naturopathie à l'environnement global en suivant une logique d'interpénétration ${ }^{23}$. Cet article a donc pour objet de répondre à la question suivante : comment le passage de contre-espaces à des espaces alternatifs témoigne et rend possible l'évolution d'une discipline qui va d'une posture orthodoxe, rurale et religieuse aux contours flous de la santé naturelle?

\section{Naturopathie des origines et retour à la nature}

\section{Tournant contemporain de la naturopathie}

La naturopathie contemporaine se trouve à un tournant, qui se manifeste en premier lieu par une crise de sa nomination juridique et thérapeutique. En effet, la naturopathie française pâtit d'une absence de définition institutionnelle. Ignorée par l'État, peu connue en tant que thérapie en dehors du cercle des initiés, la naturopathie est principalement définie par certains acteurs de la discipline, qui évoluent dans le cadre de centres de formation et sont les auteurs d'ouvrages spécialisés. Puis, à l'instar d'autres médecines douces, la naturopathie traverse actuellement une période de flou concernant la catégorie qui la caractérise. Refusant les termes de médecines douces, alternatives, naturelles, adressés par les acteurs extérieurs, elle propose des catégories lexicales qui ne sont pas reconnues renforçant par là l'asymétrie des rapports de force. La pratique de la discipline ne correspond pas non plus aux catégories existantes : il ne s'agit pas exactement de bien-être ou de santé, plus tout à fait d'alimentation, et encore moins seulement de santé préventive; aucune notion ne suffit à capter les pratiques et les représentations des acteurs de la naturopathie. Cette crise touche également le sens même du terme naturopathie : aucune école n'est en mesure de donner une définition commune de cette thérapie. Cette base uniforme dissimule la pluralité des formes de la discipline, développée au sein de centres

$\overline{23}$ Nilüfer Göle, Interpénétrations. L'Islam et l'Europe, Paris, Gaalade, 2005. 
de formation divers. Ces institutions diffèrent principalement par les techniques à l'aide desquelles elles accompagnent les individus vers le renforcement de leur force vitale. Si l'alimentation reste, dans toutes les définitions, la base de la naturopathie, le nombre des autres techniques thérapeutiques employées et leur importance varie ${ }^{24}$.

La naturopathie existe en France sous sa forme contemporaine depuis les années 1930; pourtant, elle aura attendu le début du $\mathrm{XXI}^{\text {e }}$ siècle les mutations qui, intervenant dans différentes branches de la discipline, représentent autant de tentatives pour projeter cette thérapie hors des contre-espaces auxquels elle était confinée. Deux séries d'événements attestent du changement très récent survenu dans ce domaine, et qui l'ancre dans la modernité. Habituellement, les médecines douces sont l'objet de travaux en anthropologie ${ }^{25}$ qui les étudie d'un point de vue idiosyncrasique, en tant que figures résurgentes du passé ou reléguées à la marginalité. Or, la naturopathie actuelle, résolument moderne, ne représente absolument pas une figure de la tradition mais plutôt un hybride, un entre-deux, en utilisant les éléments de la médecine hippocratique pour proposer une lecture critique de la société occidentale moderne.

En premier lieu, la parution conjointe en 2008 de deux ouvrages, l'un d'ordre historique, L'Encyclopédie historique de la naturopathie, l'autre d'ordre juridique, Le Livre blanc de la naturopathie, rédigés par les professionnels de la FENAHMAN

24 La Fédération Française de Naturopathie (FENAHMAN), qui regroupe trois des principales écoles parisiennes dédiées à la discipline, la définit comme suit : "La naturopathie rassemble les pratiques issues de la tradition occidentale et repose sur les dix agents naturels de santé fondés sur le principe de l'énergie vitale de l'organisme. Elle vise à préserver et optimiser la santé globale de l'individu, sa qualité de vie, ainsi qu'à permettre à l'organisme de s'auto-régénérer par des moyens naturels ". Les techniques auxquelles cette définition fait référence sont les suivantes : l'alimentation ou hygiène nutritionnelle, la psychologie ou hygiène neuropsychique, les exercices physiques ou hygiène musculaire ou émonctorielle, mais aussi l'hydrologie, les techniques manuelles, la réflexologie, les plantes ou phytologie, les techniques énergétiques ou magnétisme et les techniques vibratoires.

25 Marie-Pierre Estager, La Naturopathie en Aquitaine, thèse d'anthropologie, Université Bordeaux II-Victor Segalen, 2001. 
apparaît comme un véritable travail de retour aux sources. Ces ouvrages qui s'adressent aux consommateurs et aux pouvoirs publics soulignent le développement de nouvelles stratégies de conquête du public. Dans un second temps, la création de nouveaux espaces et de nouvelles professions (praticien de santé naturopathe, directeur-gestionnaire de centres de soins) accompagne le développement de lieux dédiés à la naturopathie, ancrés dans l'espace urbain, qui cherchent à attirer une nouvelle clientèle.

L'évolution de la naturopathie peut se mesurer à la manière dont chaque école s'approprie l'héritage du " père fondateur ". Partant d'une source unique, l'oeuvre de Pierre V. Marchesseau largement inspirée par l'ésotérisme et la mouvance New Age, la naturopathie actuelle est plongée dans un conflit de reconnaissance de ses origines. L'étude de la construction d'une mémoire spécifiquement naturopathique, prenant ses distances par rapport à la mouvance New Age, constitue la première clé de compréhension de l'évolution de la discipline.

2. Une discipline fondée sur une personnalité charismatique

Née aux États-Unis au début du $\mathrm{XX}^{\mathrm{e}}$ siècle, la naturopathie française s'inspire du mouvement hygiéniste européen, et plus spécifiquement allemand. Elle a été rapportée, traduite et complétée en France dans les années 1930 par le thérapeute Pierre V. Marchesseau ${ }^{26}$, désigné à l'unanimité comme le " père fondateur " de la naturopathie. Afin d'éclairer la filiation entre la naturopathie, telle qu'elle a été introduite en France par Pierre V. Marchesseau dans les années 1940, et la mouvance New Age, il faut remonter aux sources de la discipline. C'est donc dans le récit construit autour du personnage et dans les livrets rédigés, publiés, et commercialisés par lui à partir des années 1930, et jusque dans les années 1980, que j'ai pu découvrir ces éléments

26 En 1902, le médecin d'origine allemande Benedikt Lust fonde à New York l'École américaine de naturopathie (American School of Naturopathy). Cette date est considérée comme le point de départ d'une formulation moderne de la discipline. Néanmoins, les auteurs de la thérapie inscrivent cette médecine naturelle dans la filiation directe de la médecine hippocratique. 
tus ou ignorés par la plupart des enquêtés, quoiqu'existant toujours en filigrane dans l'imaginaire véhiculé par la discipline. Se posant comme le créateur d'une thérapie nouvelle, Pierre V. Marchesseau réalise dès le départ un schisme en donnant à la naturopathie la spécificité d'une discipline thérapeutique indépendante des courants ésotériques desquels elle est issue. Tout en conservant la structure religieuse de la mouvance New Age, le père fondateur a initié un détachement qui se traduit aujourd'hui par une rationalisation de la discipline. Le père fondateur, s'il n'est pas à l'origine d'une secte - les fondements de la spiritualité naturopathique ne différant guère de la mouvance New Age n'en assure pas moins la fonction de personnalité charismatique. En premier lieu parce qu'il est, d'après ses disciples, auteur de guérisons présentées comme miraculeuses, parce que venant soigner des pathologies extraordinaires. Ainsi, Marchesseau aurait sauvé son beau fils de la leucémie uniquement avec des méthodes naturopathiques. Le mythe de Pierre V. Marchesseau en tant que personnalité charismatique est renforcé par des éléments mystérieux, rendant la comparaison avec l'idéaltype d'autant plus fidèle. Son savoir serait dû à la découverte d'une malle, cachée dans le grenier de son père, de laquelle il aurait tiré des enseignements naturopathiques mais aussi mystiques. Les origines de ce corpus seraient donc inconnues; à mots couverts, les enquêtés entendent qu'il s'agirait d'une forme de révélation.

Dans la droite lignée de l'occultisme et de l'ésotérisme, la naturopathie orthodoxe et originelle se construit donc, à travers les écrits de son fondateur, dans l'ombre d'une science qui ne reconnaît pas les travaux $\mathrm{du}$ " père de la naturopathie ». Dans ses écrits, Pierre V. Marchesseau tente de prouver la véracité de sa démonstration relative aux débuts de l'humanité en s'appuyant sur deux éléments : la preuve par l'histoire et la preuve par l'universel. Singeant des méthodes plus académiques sur des sujets non reconnus par le monde scientifique, comme l'existence des Atlantes ou l'astrologie - qu'il nomme " science astrologique " - Pierre V. Marchesseau invoque l'authenticité d'un fait en rappelant sa genèse, "à l'aube de la conscience de l'humanité ", ou son 
importance dans «les textes sacrés de l'Indouisme ${ }^{27}$ ». Il propose en contrepartie de la science académique une vision alternative du christianisme, enrichie d'une dimension scientifique, intégrant un mode de vie naturopathique et sain, dans lequel la démarche expérimentale aurait pour objectif de souligner la véracité de la parole divine. À travers cette production littéraire, Pierre V. Marchesseau opère un schisme, non par rapport à la société, mais relativement à la mouvance New Age et au mode de vie communautaire, dont il critique fortement ce qu'il nomme le «sensualisme ".

\section{Diversification des sources et différenciation des courants}

1. Réappropriation des sources historiques de la naturopathie

Si la grande majorité des chefs de file actuels de la naturopathie ont été confrontés à la personnalité charismatique de Pierre V. Marchesseau dans les années 1960-1970, puisqu'ils ont été formés par lui, seule une partie d'entre eux continue à pratiquer cette technique de soins à partir de la base spirituelle et thérapeutique mise en place par le maître. L'Encyclopédie historique de la naturopathie, qui souligne la pluralité des courants et pensées ayant nourri la discipline, reflète un nouveau courant qui attire chaque année un nombre croissant de clients et d'élèves. Désireux de sortir du cadre circonscrit des médecines alternatives, et tentant d'adapter la posture holistique à la société de masse, ce nouveau courant qui traverse la naturopathie à partir des années 1990 constitue un deuxième moment dans l'histoire de la discipline. S'il continue à coexister avec une naturopathie orthodoxe majoritairement rurale, il devient néanmoins la vitrine officielle de cette posture thérapeutique. C'est à partir de la réappropriation des sources et des textes produits par Pierre V. Marchesseau que le processus de différenciation actuel, divisant la naturopathie orthodoxe et la naturopathie urbaine, se réalise.

La première tendance, dite orthodoxe directement inspirée du

$27 \quad$ Pierre-Valentin Marchesseau, Jésus-Christ naturopathe, un enseignement passé sous silence dans le Nouveau Testament, Les petits précis de l'humanisme biologique, 1936. 
rôle du fondateur, est représentée par l'école et centre de soins Univers, dirigée par M. Dargère ${ }^{28}$. Ce dernier raconte Marchesseau à la manière d'un mythe incarné, fondateur de la "philosophie vitaliste ", ou " humanisme biologique ", reposant sur les " trois écologies, interne, externe, et sociale ", par lesquelles il explique la naturopathie. L'incapacité du monde médical à faire face aux problèmes de santé actuels, la " généralisation de la folie » et la crise écologique sont pour lui autant de symptômes annonçant la fin d'une ère, et le début d'une autre, dans laquelle régnera l'humanisme biologique. À mots couverts, M. Dargère fait référence à la fin du monde, théorisée par la mouvance New Age, devant aboutir au passage à l'ère du Verseau ${ }^{29}$. Considérant la société au stade de "la dégénérescence ", il explique longuement l'interrelation entre le milieu naturel et le milieu social, et présente le changement que la planète est en train de vivre comme le signe du déclin de l'humanité : "à partir du moment où la nature change, l'espèce qui est dedans va disparaître, dégénérer puis disparaître".

Si l'Encyclopédie historique reconnaît également à Pierre V. Marchesseau son action dans la première étape de la rationalisation de la naturopathie, à travers cette capacité à résumer et à homogénéiser des savoirs glanés aussi bien dans la médecine pharaonique que dans la méthode Coué, il n'en demeure pas moins qu'il limite le rôle du biologiste autoproclamé à celui d'un traducteur, rassemblant et homogénéisant une discipline oubliée, directement reliée à la médecine hippocratique. Cette volonté d'exégèse devient un mouvement renaissant, à la lumière du travail effectué par la Fédération. En effet, la rédaction très rapprochée du Livre blanc de la naturopathie, et de l'Encyclopédie historique de la naturopathie a pour objectif d'asseoir les bases de la discipline, auprès des professionnels, mais également auprès des autorités et du grand public. Jusque là, les références biblio-

\footnotetext{
28 À l'exception des noms précédés du titre - Ms. Dargère, Kieffer et Passebecq - relatifs aux chefs de file des différents courants de la naturopathie, les prénoms des enquêtés ont été remplacés par des pseudonymes.

29 Marie-Jeanne Ferreux, Le New Age, ritualité et mythologies contemporaines, Paris, L'Harmattan, 2003.
} 
graphiques en matière de naturopathie portaient essentiellement sur des aspects circonscrits de la discipline, liés à une pathologie, à une technique ou à un organe. Même dans les livrets de Pierre V. Marchesseau, les documents de nature ésotérique à proprement parler se distinguent à grand peine dans la foule des thérapies concrètes. L'enjeu actuel semble être de montrer à la société tout entière que la discipline est capable de s'intégrer au champ de la santé publique française. De technique de soins spécifique, la naturopathie devient une posture thérapeutique. Parlant du Livre blanc, l'un de ses auteurs déclare :

C'est l'outil, c'est l'interface pour moi entre la profession et la grande presse, et surtout, surtout c'est la carte de visite pour aller voir le Ministère, c'est l'outil indispensable pour être recevable... un Livre blanc, c'est un manifeste, au bénéfice d'une cause ou d'une profession.

Mais ce travail de compilation des données et de retour aux sources ne se limite pas à l'œuvre des chefs de file de la profession. De manière individuelle, certains thérapeutes tentent de participer à cet effort. Ainsi, Patrick, fils d'un naturopathe orthodoxe, commercialise sur les salons parisiens et provinciaux les cahiers de naturopathie rédigés par son père, remis au goût du jour et épurés de leurs aspects les plus ésotériques. De la même façon, Christophe se propose d'entamer un travail de réécriture et de réédition des livrets de Marchesseau, alors que $M$. Dargère les commercialise tels quels et sans tabous sur tous les salons et par correspondance.

2. Naturopathie rurale, naturopathie urbaine : les espaces de la naturopathie

Au-delà des travaux d'écriture consacrés à la naturopathie, remonter le fil de la discipline à partir de l'espace des salons bioéthiques m'a permis de rencontrer deux types de naturopathies, rurale et urbaine. Dans le premier cas, le rapport des acteurs à la nature contraste avec le mouvement New Age et communautaire dans les années 1970, tel que le décrit Paul Heelas $^{30}$, ou encore Danièle Léger et Bernard Hervieu dans Le

30 Paul Heelas, The New Age Movement, op. cit., p. 5. 
Retour à la terre ${ }^{31}$, écrit en 1979. Dans cet ouvrage, les auteurs mettent en lumière les répercussions du passage à un mode de vie rural de jeunes urbains, suite au mouvement de mai. Également persuadés que le printemps de l'année 1968 ne constitue pas le point de départ des utopies New Age, les auteurs décrivent cet élément déclencheur comme un " moment de rencontre entre l'occultisme, le New Age, et tous les mouvements religieux essayant de relier science et théologie, et l'action sociale d'une société civile qui progressivement se désinstitutionnalise et se syncrétise ${ }^{32}$ ", qui agit comme les prémices d'un déplacement géographique, tourné vers une ruralité jusque là fantasmée. Les auteurs citent Jean Séguy, pour qui les nouveaux arrivants ont pu trouver, dans le contexte d'une campagne désertée, ce qui « fait le mouvement même de l'utopie : un appel au passé, qu'on reconstitue souvent en un âge d'or magnifié contre un présent qu'on rejette, en vue d'un avenir radicalement autre ${ }^{33}$ ". En inversant le sens de l'exode rural, survenu vingt ans plus tôt, les jeunes ne recherchent pas tant une réponse à des aspirations écologiques et agricoles encore floues qu'une alternative à leur mode de vie qui s'exprime avant tout par le décalage de la territorialité : « extériorité géographique par rapport à l'espace urbain et industriel, qui est aussi l'espace où se déploient les luttes politiques et syndicales sérieuses ${ }^{34}$ ». Ainsi, le retour à la nature consisterait à donner lieu à une utopie glorifiant un âge d'or perdu et que l'on voudrait retrouver. Davantage qu'un déplacement géographique, cette délocalisation représente une projection de soi vers une nature étrangère à la société occidentale moderne et urbaine. En rupture avec le mouvement hygiéniste dont l'urbanisme consistait à concilier ville et campagne, le retour à la terre des années 1970 représente un rejet de la Metropolis à l'origine de tous les maux, formulé dès le début du siècle, mais moteur d'une action de masse à partir de 1968. Le retour à la terre est la condition sine qua non de la contre-culture, la «muta-

\footnotetext{
31 Bernard Hervieu et Danièle Léger, Au fond de la forêt..., op. cit., p. 4.

32 Ibid., p. 32.

33 Ibid., p. 4.

34 Ibid., p. 47.
} 
tion irréversible des structures économiques, des règles de vie, des attitudes mentales ${ }^{35}$ ", secrétant des modèles et des références propres.

Comme les autres acteurs affiliés aux mouvements contreculturels, les naturopathes suivront ce mouvement. M. Kieffer ${ }^{36}$, ému, raconte à ses élèves sa rencontre avec le célèbre mystique Lanza del Toro, dans la communauté duquel il fera quelques séjours avec sa femme. Certains naturopathes faisant figure de chefs de file dans la discipline - M. Dargère et M. Passebecq ${ }^{37}$, mais aussi Philippe ${ }^{38}$, propriétaire d'un magasin à Paris et directeur d'un centre de formation dans les environs de SaintÉtienne, Michel ${ }^{39}$ ou Christophe ${ }^{40}$, praticiens de santé installés à la campagne - ont fait le choix de maintenir un mode de vie rural, dans une démarche de symbiose avec la nature. En dehors des moments marqués par l'effervescence des salons nature et santé comme le Salon Marjolaine, ils assurent leurs formations et leurs consultations depuis les villages où ils exercent. Le cadre où se déroulent les enseignements et les consultations constitue d'ailleurs un atout majeur incitant la clientèle à venir. Cette

35 Edgar Morin (dir.), La Croyance astrologique moderne, diagnostic sociologique, Paris, l'Âge d'Homme, 1984, p. 31.

36 Président de la Fédération française de naturopathie et directeur du centre de formation CENATHO, situé à Paris, Daniel Kieffer est le chef de file de la naturopathie urbaine. Il est à l'origine du mouvement de reconnaissance juridique de la discipline et a publié en 2008, outre une importante bibliographie portant sur les techniques de soins de la naturopathie, Le Livre blanc de la naturopathie ainsi que L'Encyclopédie historique de naturopathie.

37 Ancien collaborateur de Pierre V. Marchesseau, Michel Passebecq exerce en région PACA. Il est l'une des figures emblématiques de la naturopathie historique.

38 Bien qu'installé à Paris, Philippe, ancien ingénieur agronome formé à la naturopathie au Québec dans les années 1980, est une figure de la naturopathie rurale. L'essentiel de son activité se déroule dans le magasin d'alimentation biologique et de cosmétiques bio qu'il loue à Paris et où il pratique également des soins.

39 Ancien employé dans l'industrie pharmaceutique, Michel exerce la naturopathie vitaliste de Pierre V. Marchesseau en zone rurale, en région parisienne.

40 Autodidacte en naturopathie, Christophe se définit comme «magnétiseur et naturopathe ". Il a créé en 2006 une entreprise de commercialisation de produits " naturels " qu'il promeut sur les salons Nature et Santé, tout en maintenant une activité de thérapeute en zone rurale, en région parisienne. 
branche de la naturopathie, autoproclamée orthodoxe, applique donc la philosophie vitaliste de Pierre V. Marchesseau à la vie quotidienne. D'autres naturopathes prennent la défense de cette hygiène de vie considérée comme étant à la fois plus spirituelle et plus ascétique, qui se tient volontairement à l'écart des pratiques de consommation de masse, et n'hésitent pas à insister lors des entretiens, sur leurs expériences «transpersonnelles » ou leurs croyances réincarnationistes :

Le naturopathe est vitaliste. Le vitalisme considère que la vie vient habiter la matière sous certaines conditions physico chimiques... A contrario, le matérialiste considère que la vie est issue de la matière... D'un côté le matérialiste considère que la vie est issue d'un hasard, de l'autre côté le vitaliste considère que la vie est organisée, structurée, cohérente, a une intelligence du vivant, et que le hasard n'existe pas. Et que l'être humain fait partie de ce tout, et le job du praticien ça va être de comprendre cette intelligence de vie pour l'accompagner (Christophe, naturopathe de 38 ans, installé en Provence).

3. Passage de la confrontation conflictuelle à l'interpénétration douce

Le passage de l'ésotérisme et de la ruralité à l'exotérisme et au territoire urbain est notamment rendu possible par l'évolution des formes de la confrontation entre cette posture, minoritaire dans le champ de la santé, et l'espace public. Alors qu'au temps de Pierre V. Marchesseau la naturopathie était en conflit ouvert avec la médecine et l'État, la décennie 2000 marque les prémices d'une confrontation plus pacifique ${ }^{41}$. Bien qu'ils évoquent souvent les fondements vitalistes de la naturopathie dans le cadre de conférences ou de présentation de centres de formation, les membres de la FENAHMAN, chefs de file de la discipline, membres du corps professoral ou anciens élèves bien intégrés au

$41 \quad$ Ainsi, dans les années 1970, la naturopathie de Pierre V. Marchesseau était très critiquée par les pouvoirs publics. M. Dargère raconte une rencontre lors de laquelle M. Poniatowski, alors Ministre de la santé, aurait raillé le «père fondateur " en ces termes : "M. Marchesseau, vous ne fumez pas, vous ne buvez pas, mais vous êtes un homme très dangereux pour la société ». 
réseau naturopathique, relèguent le père fondateur au rôle de traducteur et de catalyseur de la naturopathie en France.

Cet adoucissement de la confrontation est notamment dû à la progression des techniques de soins considérées comme normales par les naturopathes eux-mêmes. Les naturopathes orthodoxes entendent maintenir l'enseignement de Pierre V. Marchesseau comme fondement unique de la naturopathie, regrettant la dislocation progressive du vitalisme et la perte de l'usage de techniques considérées comme drastiques, telles que les mono diètes, les jeûnes et les lavements. Ainsi, Damien ${ }^{42}$, naturopathe orthodoxe, établit une hiérarchie entre les différents types de naturopathie, la meilleure restant, selon lui, la plus proche de l'originelle. Créateur, selon lui, d'un nouveau principe de société, dans lequel la naturopathie est avant tout une philosophie, correspondant à une science et à une technique, Pierre V. Marchesseau, inventeur du vitalisme, aurait considéré que la vie vient habiter la matière et non l'inverse :

$\mathrm{Tu}$ as des naturo qui sont très hygiénistes... qui ont une pensée très alimentation, et, sur le jeûne, sur une approche très peu, très peu, très peu interventionniste $[. .$.$] qui vont aller sur des méthodes hygiénistes...$ pures... c'est-à-dire c'est l'utilisation, c'est le lavement, c'est le jeûne, c'est des mono-diètes, c'est... l'utilisation de l'eau froide... des méthodes on pourrait dire entre guillemets, très orthodoxes.

Les naturopathes urbains, quant à eux, optent pour des techniques de soins plus souples. De nombreux praticiens de santé avouent leurs petites faiblesses, notamment alimentaires. Yaël s'exprime ainsi : " quand je vois dans les salons tous les gens qui sont vraiment très pâles et très... intégristes... Moi, je suis contre l'intégrisme ». Cette enquêtée, ancienne metteur en scène de théâtre, fait partie de cette nouvelle population, dont le mode d'action est caractéristique d'un passage de la confrontation conflictuelle à l'interpénétration douce. Cette nouvelle clientèle des écoles et des produits, qui constitue la raison d'être de ce travail, témoigne de l'attrait de nouvelles catégories de population pour une discipline du corps et de l'esprit en demande d'un autre

42 Damien est un jeune naturopathe de trente ans récemment formé au CENATHO et exerçant la naturopathie à son domicile. 
mode de vie. Elle compose aujourd'hui l'essentiel de la naturopathie urbaine. Dans le cas des thérapeutes s'inscrivant dans une transmission naturopathique, on observe le même passage d'une compréhension ascétique de la discipline à une appréhension confortable d'un mode de vie plus naturel. Le témoignage de Patrick, lui-même fils de naturopathe orthodoxe revenu à la discipline après dix ans de rejet, illustre cette évolution marquant le passage de techniques de soins aujourd'hui qualifiées de strictes ou d'ascétiques vers un mode d'alimentation et un mode de soins perçus comme étant plus souples par les professionnels : " mon père, mon père qui était très strict en naturopathie, dans la vie en général, c'était quelqu'un de très strict ". Lui prône une naturopathie à la fois plus souple dans ses méthodes et dans son hygiène de vie et plus médicalisée et moins spirituelle, du moins en apparence. Refusant d'écrire dans des revues trop connotées New Age, comme le mensuel Soleil Levant, il consacre une partie de son temps à la co-rédaction d'un ouvrage comparant l'efficacité des médecines douces et de la biomédecine. D’aspect bien portant, contrastant avec la maigreur ascétique des naturopathes plus orthodoxes, il avoue ses écarts alimentaires tout en sirotant son café, boisson pourtant peu recommandée par les naturopathes. De la même façon, plus franchement que ses confrères, qui taisent de tels épisodes, Bruno, ancien assistant de Pierre V. Marchesseau et membre privilégié du clan FENAHMAN, évoque sa propre évolution depuis les années 1980 où il accompagnait le " maître " dans tous ses déplacements, l'assistant pour les activités liées à la naturopathie comme pour les réunions ésotériques, imposant à ses enfants une éducation sévère à l'extrême : "J'ai eu mes premiers enfants, mes premiers jumeaux, là... que j'ai élevés d'une manière naturopathique, mais alors plus, plus, plus... rires... style Marchesseau ". Aujourd'hui, Bruno, enseignant au CENATHO, a adopté une pratique moins rigide en termes de choix et de prescriptions alimentaires, et a laissé de côté des techniques de soins comme le jeûne ou les lavements. 


\section{Les nouveaux enjeux de la naturopathie urbaine}

\section{La sortie de l'ésotérisme ou la voie de la légalisation}

Invoquant sans cesse une rationalité scientifique dans les sillons de laquelle elle cherche à s'inscrire, la naturopathie urbaine rejette l'utopie d'une société individualiste comme les éléments religieux du corpus New Age. Elle se place dans un processus de sécularisation qui relègue le religieux au domaine privé, du moins en partie. Loin d'affirmer sa toute puissance, elle cherche à se définir avant tout par ses limites. L'expression latine non nocere, ne pas nuire, pourrait en être la devise. De la même façon, les naturopathes urbains reconnaissent à l'unanimité l'importance de la biomédecine dans les situations d'urgence. En effet, l'un des éléments permettant de différencier la naturopathie rurale, fidèle aux principes développés par Pierre V. Marchesseau qui s’opposait au recours à la médecine, et la naturopathie urbaine, réside justement dans le discours des enquêtés sur le rapport à la médecine allopathique. Plus question de soigner une leucémie en vivant en autarcie avec le malade, ni d'éliminer les antibiotiques pour guérir les maladies infectieuses infantiles. Les naturopathes se réservent plutôt une fonction de prévention et d'accompagnement en cas de pathologies lourdes, et un travail d'accompagnement de la vie quotidienne en cas d'absence de maladie grave. Enfin, la naturopathie contemporaine se fixe des limites dans sa narration de la discipline. Ne s'occupant plus de décrire les conditions d'existence des Atlantes ou la disparition $\mathrm{du}$ continent $\mathrm{Mu}$, laissant de côté le fantasme d'une hygiène de vie liée à la vie rurale, elle se nourrit de son passé avec la prétention d'être internationalement reconnue comme la médecine traditionnelle occidentale du futur. Autoproclamée « melting-pot de toutes les médecines orientales et occidentales ${ }^{43}$ " reposant sur l'ensemble des " techniques ancestrales ", cette médecine naturelle puise ses sources dans la médecine hippocratique, qu'elle voudrait remettre au goût du jour.

$\overline{43}$ Entretien avec la secrétaire de l'OMNES, Organisation professionnelle des naturopathes. 
Tout en maintenant une partie de ces prescriptions en matière d'hygiène de vie, la naturopathie, dans sa définition d'elle-même, dans ses ouvrages de référence comme dans le discours de ses professionnels, est déjà sortie de l'ésotérisme d'un cercle d'initiés limité pour l'exotérisme d'une pratique de plus en plus assumée. Cette évolution peut être mise en parallèle avec le cas de l'astrologie moderne, étudiée par Edgar Morin :

L'auteur exotérique moderne regarde volontiers vers l'avenir, qu'il souhaite différent du passé, l'ésotériste reste en revanche tourné vers le passé et aspire à en restituer la précieuse forme originelle. La négation du moi, la revendication d'impersonnalité, le rejet de l'originalité, l'attribution de l'enseignement à un lointain maître, sont les moyens conventionnels du genre où la transmission est plus prisée que l'invention, car les doctrines secrètes, les enseignements spirituels et les pratiques rituelles ne doivent pas provenir d'un homme, mais d'une révélation divine à laquelle une sombre nuit des temps convient parfaitement comment lieu d'origine ${ }^{44}$.

2. De la naturopathie comme discipline à la santé naturelle aux contours flous

Avançant à grands pas, la naturopathie urbaine contemporaine est actuellement en tension. Elle est partagée entre la volonté de démocratiser la discipline en la sécularisant et la crainte de perdre les fondements et les contours de celle-ci à travers une posture thérapeutique édulcorée. Fruit d'une politique centralisée de visibilité sur de nouveaux espaces, menée par les organisations actives de la discipline, ou sur des espaces attirant davantage de gens, ou conséquence implicite d'une sécularisation générale du New Age qui se confronte à ce paradigme inédit, le nouvel enjeu de la naturopathie est de parvenir à rester fidèle à elle-même tout en se projetant dans un futur marqué par la reconnaissance.

Alors que cette seconde génération de naturopathes consolide son chemin, sinon vers la légalisation, du moins vers la connaissance et la reconnaissance tacite de la discipline par la société, une troisième génération de praticiens et de clients est actuellement en cours de formation. Ce constat nous donne un premier élément

44 Edgar Morin (dir.), La Croyance astrologique moderne, op. cit., p. 28. 
permettant de confirmer l'hypothèse selon laquelle la naturopathie organise sa sortie de l'ésotérisme en s'insérant sur d'autres espaces.

\section{Conclusion}

En cherchant à redéfinir sa genèse à partir d'une pluralité de sources, remettant notamment en question la nécessité de l'ascétisme et du communautarisme, la naturopathie gagne de nouveaux espaces et, par conséquent, de nouveaux publics. Ce double processus de distanciation/assimilation s'accompagne d'une visibilité croissante de la discipline sur les espaces alternatifs du commerce, des médias et de la formation à travers les salons, les magasins bio, les centres de soins, etc., à la création desquels elle participe, et qui attirent un nombre croissant de clients. Bien qu'étant peu connue du grand public, la naturopathie se développe sur un marché du bien-être et de l'alimentation et de la santé naturelles de plus en plus dense, complexe et lucratif.

Le combat actuel des naturopathes urbains est donc celui de la reconnaissance juridique de leur discipline comme thérapie. Le passage d'une confrontation conflictuelle, caractéristique de la contre-culture à une interpénétration douce est celui de l'évolution d'une conception révolutionnaire et utopiste des mouvements sociaux à une vision réformatrice de l'action sociale, proposant un mode de vie. Cette refonte des frontières entre biomédecine et médecines douces, entre combat écologique et lutte pour la reconnaissance des thérapies alternatives, participe de la création d'une "santé naturelle " aux contours flous, imprégnant les espaces de la nébuleuse écologico-environnementale ${ }^{45}$.

$\overline{45}$ André Micoud, «De l'expert-militant à l'être vivant sensible », dans Marcel Boyer, Guy Herzlich et Bruno Maresca (dir.), L'Environnement, question sociale. Dix ans de recherches pour le ministère de l'Environnement, Paris, Odile Jacob, 2001. 


\section{Bibliographie}

Bailly, Eliane, Les Ostéopathes face à la contrainte institutionnelle : genèse d'une professionnalisation, thèse de sociologie, Université de Nantes, 2006.

Descola, Philippe, Par-delà nature et culture, Bibliothèque des sciences humaines, Gallimard, Paris, 2005.

Diaz Pedregal, Virginie, Le Commerce équitable dans la France contemporaine, Paris, L'Harmattan, 2008.

Estager, Marie-Pierre, La Naturopathie en Aquitaine, thèse de doctorat en anthropologie, Université Bordeaux II-Victor Segalen, 2001.

Faure, Olivier, Histoire sociale de la médecine (18 ime-20 ime siècle), Paris, Anthropos, 1994.

Ferreux, Marie-Jeanne, Le New Age, ritualité et mythologies contemporaines, Paris, L'Harmattan, 2003.

Grisoni, Anahita, Sous les pavés la terre : entre culte du bien-être et nouveaux métiers : la naturopathie en transformation à la conquête de l'espace public, thèse de doctorat en sociologie, Paris, École des hautes études en sciences sociales, 2011.

Göle, Nilüfer, Interpénétrations. L'Islam et l'Europe, Paris, Gaalade, 2005.

Heelas, Paul, The New Age Movement, Londres, Blackwell Publishers, 1996.

Hervieu, Bernard et Danièle Léger, Le Retour à la nature, au fond de la forêt... l'État, Paris, Seuil, 1979.

Lamine, Claire, Les Intermittents du bio, pour une sociologie pragmatique des choix alimentaires émergents, Paris, La Maison des Sciences de l'Homme, 2008.

Lenoir, Frédéric et Nathalie Luca, Sectes, mensonges et idéaux, Paris, Bayard, 1997.

Micoud, André, « De l'expert-militant à l'être vivant sensible », dans Marcel Boyer, Guy Herzlich et Bruno Maresca (dir.), L'environnement, question sociale. Dix ans de recherches pour le ministère de l'Environnement, Paris, Odile Jacob, 2001, p. 121-134.

Morin, Edgar (dir.) La Croyance astrologique moderne, diagnostic sociologique, Paris, l'Âge d'Homme, 1984.

Pace, Enzo, "Le retour du sacré », dans James Andrew Beckford et Danièle Hervieu-Léger (dir.), Religion et Écologie, Paris, Le Cerf, 1993, p. 197-213.

Pleyers, Geoffrey (dir.), La Consommation critique. Mouvements pour une alimentation responsable et solidaire, Paris, Desclée de Brouwer, 2010. 
Rudolf, Florence, Le Climat change... et la société?, Éditions Engagé-e-s, Paris, 2008.

Sfez, Lucien, La Santé parfaite, Paris, Seuil, 1995.

Tétard, Gilles, Le Sang des fleurs, une anthropologie de l'abeille et du miel, Paris, Odile Jacob, 2005.

Touraine, Alain et al., La prophétie anti-nucléaire, Paris, Seuil, 1980.

\section{Bibliographie naturopathique}

Collectif FENAHMAN., Le Livre blanc de la naturopathie, Paris, Yves M. Michel, 2008.

Kieffer, Daniel, Encyclopédie historique de la naturopathie, Paris, Jouvence, 2008.

Marchesseau, Pierre-Valentin, Jésus-Christ naturopathe, un enseignement passé sous silence dans le Nouveau Testament, Les petits précis de l'humanisme biologique, 1936. 